Canadian University Music Review

Revue de musique des universités canadiennes

\title{
Le langage des tambours dans la cérémonie vaudou haïtienne
}

\section{Nicole Beaudry}

Numéro 4, 1983

URI : https://id.erudit.org/iderudit/1013900ar

DOI : https://doi.org/10.7202/1013900ar

Aller au sommaire du numéro

Éditeur(s)

Canadian University Music Society / Société de musique des universités

canadiennes

ISSN

0710-0353 (imprimé)

2291-2436 (numérique)

Découvrir la revue

Citer cet article

Beaudry, N. (1983). Le langage des tambours dans la cérémonie vaudou haïtienne. Canadian University Music Review / Revue de musique des universités canadiennes, (4), 125-140. https://doi.org/10.7202/1013900ar

(C) Canadian University Music Society / Société de musique des universités canadiennes, 1983
Ce document est protégé par la loi sur le droit d'auteur. L'utilisation des services d’Érudit (y compris la reproduction) est assujettie à sa politique d'utilisation que vous pouvez consulter en ligne.

https://apropos.erudit.org/fr/usagers/politique-dutilisation/ 


\title{
LE LANGAGE DES TAMBOURS DANS LA CÉRÉMONIE VAUDOU HAIITIENNE
}

\author{
Nicole Beaudry
}

Le présent article s'intéresse à la multiplicité des fonctions des rythmes vaudous en général et à la pluridimensionalité de leurs significations. Plus spécifiquement, nous voulons discuter de deux cas bien précis de construction rythmique reliée à des significations: le Kata, ou motif sous-jacent à toute la rythmique de la cérémonie vaudou, et le Cassé, une section contenue à l'intérieur du rythme de base dont il est spécifique. Mais auparavant, il nous faut décrire brièvement le contexte vaudou ainsi que les conditions d'enregistrement sur lesquelles nous appuyons nos dires. ${ }^{1}$

\section{Contexte vaudou}

-La religion vaudou en Haïti s'exprime principalement dans trois grands rituels, spécifiques d'occasions liturgiques précises. Le constraste entre les rituels « est défini surtout par les traits de caractère attribués aux loas " (Métraux 1958: 75) qui vont donner sa couleur à la célébration. Ainsi, le rituel Rada qu'on appelle parfois "cérémonie guinin " se déroule dans une atmosphère relativement calme alors que le rituel Petro répand une atmosphère intense, voire violente; le rituel Kongo est en général perçu comme étant raffiné, élégant, gentil et ses rythmes servent aussi à faire la cour. En général, un rituel s'adresse à une famille de loas (les divinités vaudouesques) et une cérémonie ne fait usage que d'un seul rituel; il arrive tout de même que deux (ou trois) rituels soient présents lors d'une même cérémonie.

Pendant les cérémonies, certains fidèles deviennent possédés par les loas et les tambours participent à la crise de possession; on dit du "possédé " qu'il est "monté " comme un cheval. Aidé de ses assistants, un prêtre (le houngan ou la 
mambo) dirige la cérémonie: il décide de la liturgie du jour et par conséquent, des loas que l'on doit invoquer; c'est lui qui indique aux tambourineurs quels rythmes jouer pour attirer ces loas. A cette fin, le houngan utilise aussi un support visuel, le vévé, un dessin sacré tracé sur le sol avec de la farine de maïs. Un vévé bien dessiné et des rythmes bien joués produiront l'effet désiré, c'est-à-dire la venue des loas qui se manifestent en " possédant " un ou plusieurs fidèles; un dessin ou des rythmes mal faits empêcheraient les loas de venir. ${ }^{2}$

Les rythmes de tambour assurent donc le succès d'une cérémonie vaudou, ce qui explique sans doute que cet élément du rituel soit si complexe. Au cours de cette recherche, nous avons repéré une cinquantaine de rythmes de base différents, exécutés par un tambour-maître, accompagné d'une cloche et d'un ou deux tambours d'accompagnement, selon le rituel. ${ }^{3}$ Dans le rituel Rada dont il sera ici question parce que ce sont les rythmes de ce rituel qui ont fait l'objet des transcriptions et des analyses les plus détaillées, le tambour-maître s'appelle manman et il est accompagné du hounto, du doudou et de la cloche: ogan.

La combinaison de ces quatre instruments percussifs transmet des messages, symboliques ou directs, mais précis, aux participants et aux loas. Il existe une quantité innombrable de noms pour désigner les loas qu'on peut plus ou moins regrouper en grandes familles qui varient ou se recoupent selon les régions, les traditions et les préférences locales. En général, des rythmes spécifiques ou des familles de rythmes s'adressent à des loas spécifiques ou à des familles de loas: par exemple, les rythmes des familles Yanvalou et Mahi (rituel Rada) s'adressent à Damballah ou à sa femme Aida Ouèdo.

Lorsqu'on dit "rythme" vaudou, on parle d'un principe rythmique de base avec des caractéristiques qui lui sont propres: durées, contour(s) intonatif(s), timbre(s), tempo, niveau(x) d'intensité et type de relation avec les autres instruments; chacun des rythmes porte un nom. Comme pour les loas, il y a des recoupements: par exemple, un même rythme utilisé dans deux rituels différents change de nom, mais jusqu'à quel point pouvons-nous parler de similitude? Souvent, même lorsque les durées et les contours intonatifs demeurent, les tempi et les timbres diffèrent. La cinquantaine de rythmes de base différents inventoriés jusqu'ici existe en dehors de ces recoupements et ne tient pas compte non plus de la grande quantité de variantes permises. 
Nous insistons sur le fait de la complexité de l'organisation rythmique parce qu'elle n'a jamais jusqu'ici été véritablement étudiée et comprise. En fait, un seul ouvrage a tenté, à notre connaissance, une exploration tout à fait méritoire de ce phénomène (Courlander 1960), mais cette tentative présente des problèmes que nous n'aborderons pas ici.

\section{Conditions d'enregistrement}

Une cérémonie vaudou offre peu de moments de silence; en réalité elle est extrêmement bruyante: des chants en répons (toujours à tue-tête), le grêlement des açons (hochet sacré que manipule le prêtre) et bien sûr les trois tambours et la cloche; en arrière-plan, le tintement des clochettes, des battements de mains, des cris, des gestes bruyants des danseurs et même, en ville, des bruits étrangers comme des camions et des avions. L'atmosphère est vivante, excitante et souvent joyeuse.

A travers toute cette activité, bon nombre d'indices permettent de reconnaître le rituel célébré. L'oeil remarque la forme, la construction et le nombre de tambours, le blanc des costumes radas, les danses, les vévés et tout l'attirail spécifique à ce rituel. L'oreille reconnaît les chants, les rythmes, le timbre des tambours et plus spécialement le motif rythmique Kata que fait le plus petit tambour (doudou) pendant toute la cérémonie.

A cause de la multiplicité de sons et de la grande variété de rythmes utilisés, la transcription des rythmes de tambours dans le véritable contexte d'une cérémonie devient une tâche ardue, sinon impossible. Heureusement, nous avons pu procéder à des enregistrements de chaque instrument et pour chaque rythme, en utilisant la méthode de "playback" bien connue des chanteurs de musique populaire et vérifiée avec succès sur une musique de tradition orale par Simha Arom (Arom 1976). Pour ce faire, un joueur doit entendre une partie au moyen d'écouteurs et ajuster son jeu à ce qu'il entend. Georges Rodriguez, un maître-tambourineur résidant à Montréal, a joué toutes les parties: d'abord un tambour sur une piste qu'il réécoutait ensuite en jouant simultanément la partie d'un autre tambour sur une deuxième piste; à l'écoute des deux tambours il rajoutait le troisième tambour sur la troisième piste et enfin, la cloche sur la quatrième piste. ${ }^{4}$ Grâce à ces enregistrements, on peut écouter chaque tambour isolément ou dans n'importe quelle combinaison de tambours, ce qui permet non seulement une transcription exacte, mais aussi une base solide de comparaison avec nos propres 
enregistrements faits à Port-au-Prince en 1979. ${ }^{5}$ Finalement, lors d'une autre série d'enregistrements, les tambours ont été groupés sur une seule piste alors que sur une deuxième piste, on ajoutait la partie vocale, ce qui, en passant, a permis de constater que la métrique mélodique ne correspond que rarement à la métrique des tambours. ${ }^{6}$

\section{Les fonctions des rythmes}

Dans une cérémonie vaudou, les tambours et leurs rythmes accomplissent plusieurs tâches. En voici quelques exemples marquants:

- Le rythme Sagoué appelle les fidèles au début de la cérémonie.

- Au moins un des rythmes de la famille Yanvalou s'adresse à Legba, le gardien du monde des loas et sorte d'intermédiaire entre les humains et les loas. A lui tout particulièrement, les humains demandent d'avoir accès au monde des loas dès le début de la cérémonie.

- Le açon (hochet sacré) tenu à la main par le houngan (prêtre) ou par un de ses assistants, sert à indiquer le tempo du rythme qui vient, révélant par le fait même quel rythme doit être joué et quel loa on attend.

- Le rythme Ochan souligne l'arrivée des invités de marque, des loas ou des spectateurs privilégiés.

- Les tambours excitent les loas par des effets spéciaux, les invitant à venir " monter» les danseurs, contribuant ainsi au phénomène de la transe-possession.

- On peut manifester sa désapprobation, si un loa non invité se manifeste, en jouant le rythme du loa qu'on désire voir arriver.

- L'activité intense aux tambours sert de support à une transe-possession alors qu'une interruption de cette activité rythmique doit en principe interrompre la transe.

- Une quantité de roulements (aux tambours) sert soit de transition d'un rythme à un autre, soit d'interlude en attendant que tous les participants soient prêts: par exemple, le maître-tambourineur, à la fin d'un moment particulièrement bruyant, peut avoir besoin de temps pour réajuster son écoute aux motifs constants des autres tambours ou à l'indication de tempo par le açon. 
On pourrait multiplier ces exemples, mais il est temps de discuter des Katas et des Cassés.

\section{Le Kata}

Le motif Kata constitue une sorte de pouls constant durant toute le cérémonie, quel que soit le rythme joué. Sa construction est un des indices qui sert à identifier de quel rituel il s'agit.

Le mot "kata " vient fort probablement du morphème utilisé pour le tambourinage verbal: " katap katap katap . . . », toujours prononcé avec la première syllabe courte et la seconde, longue: $\delta \oint \delta \&$. Bien qu'il soit toujours joué officiellement par le plus petit des tambours (le doudou), plus facile à repérer pour l'oreille à cause de son timbre aigu, il fait toutefois partie de tous les rythmes d'accompagnement et quelques fois même, du rythme de base.

Les motifs des tambours d'accompagnement changent peu et malgré la grande variété de rythmes de base, on ne trouve que six motifs Kata différents; on les a transcrits et comparés dans le tableau suivant:

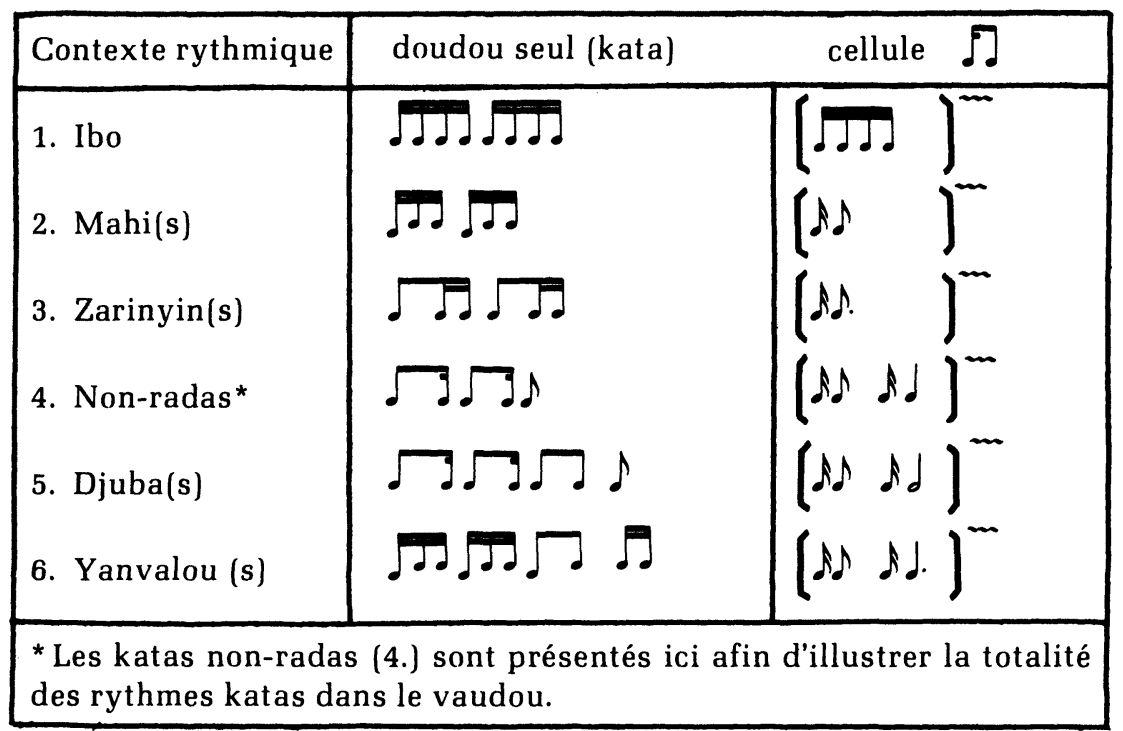

Exemple 1

Dans la colonne de gauche, on voit comment se joue le Kata lorsqu'il est isolé des autres tambours; on voit qu'il peut comporter des changements de hauteurs $\left(n^{0} 2, n^{0} 6\right)$ mais on voit aussi 
dans la colonne de droite que ce changement de hauteurs est neutralisé à l'écoute dans le contexte des quatre instruments à percussion.

Son tempo et la longueur des points d'arrêt entre les répétitions de motifs, différencient pour le pratiquant un motif Kata d'un autre ainsi que le nombre de répétitions de la cellule "Katap " $(\delta \delta)$ entre les points d'arrêt. Dans la colonne de droite de l'exemple 1 , les motifs Kata ont été placés selon un ordre de progression, c'est-à-dire qu'ils vont de l'absence de point d'arrêt à un arrêt de longueur équivalent à une blanche ( ग्रु ग्र. ... à

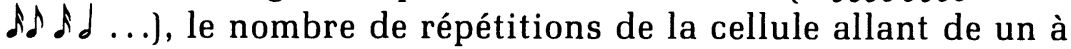
trois.

En principe, les points d'arrêt devraient correspondre à ce qui arrive dans les motifs des autres tambours mais le rythme Yanvalou simple - un des rythmes de la famille yanvalou nous offre un bon exemple de ce qui se produit en réalité:

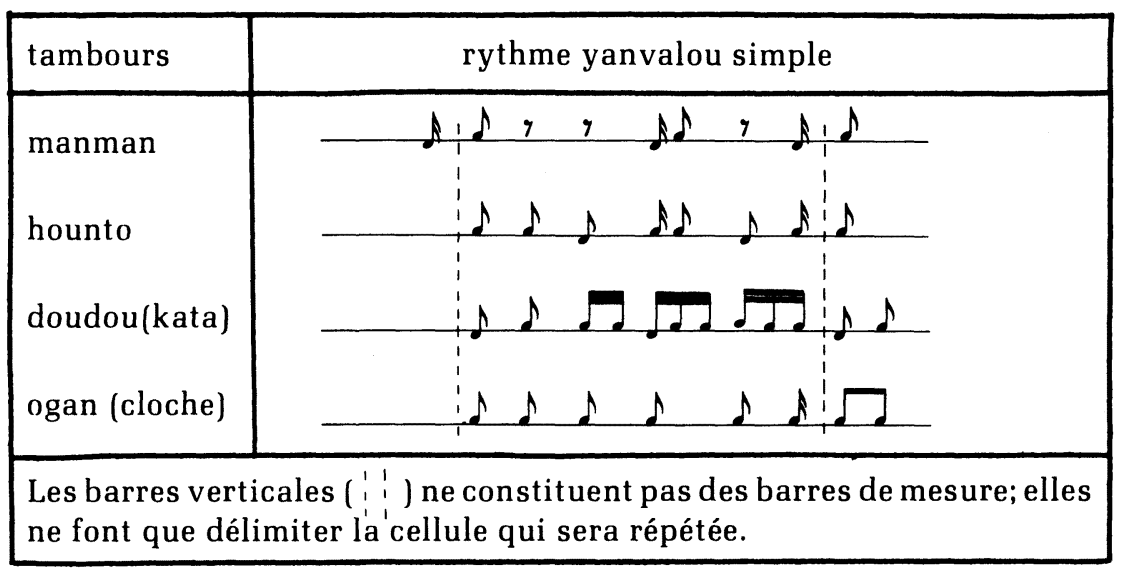

Exemple 2

Le Kata de ce rythme est celui présenté au numéro 6 de l'exemple 1 ; il faut ramarquer qu'au deuxième tambour (hounto) et à la cloche (ogan), mis à part leurs points d'arrêt identiques, la cellule « katap » ( $\$$ ) est présente, mais non de façon simultanée. L'exemple suivant isole la cellule «katap " ( $\left.)^{\prime}\right)$ du rythme Yanvalou simple et la montre telle qu'elle se présente aux différents tambours, lorsque ceux-ci sont joués ensemble: 


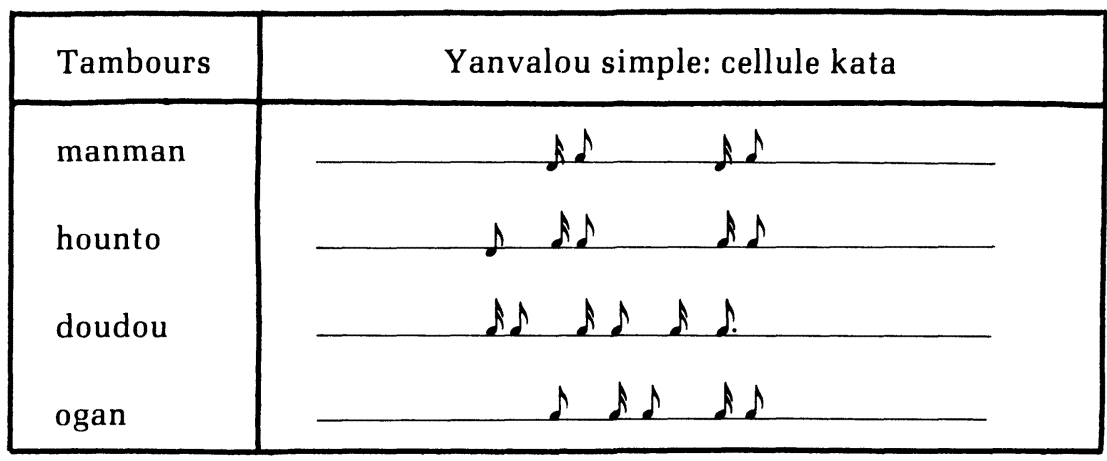

Exemple 3

En réfléchissant à la relation entre le tambour-maître (manman) et les tambours d'accompagnement, on s'aperçoit que les tambours d'accompagnements ont pour fonction de valoriser le message du tambour-maître. Par exemple lorsque le tambourmaître joue ses variations, les tambours d'accompagnement maintiennent leurs motifs réguliers et maintiennent par la même occasion l'atmosphère particulière d'une famille de rythmes. Bien sûr, les variations au tambour-maître ont leurs propres liens de parenté: en évolution constante et dispersées à travers des rappels du rythme de base, les variations ne portent même pas le nom des rythmes avec lesquels elles sont jouées. Elles sont présentes, tant pour donner au tambourineur-maître l'occasion de faire valoir sa virtuosité que pour intensifier l'atmosphère d'excitation essentielle à la venue d'un loa. De toute évidence alors, les tambours d'accompagnement renforcent l'impact du message du tambour principal en offrant un fond unifié par la répétition régulière commune à tous les tambours et constamment présente, de la cellule Kata, des motifs et de la structure rythmique.

\section{Le Cassé}

Examinons un autre type de construction rythmique. En français et en créole, le mot "cassé " veut bien dire "brisé». Aussi lorsqu'on entend un Cassé, on est frappé par l'effet de brisure dans la régularité du rythme, causée par l'intensification soudaine du volume, l'effet de syncope des nouveaux motifs et l'utilisation insistante d'un son qui porte aussi le nom de cassé, très haut et très fort. 
Les rythmes Cassés ou "changing rhythms" (Jones 1959) apparaissent plusieurs fois, soit au début, au milieu et à la fin des rythmes. A très peu d'exceptions près, tous les rythmes ont une section Cassé qui leur est spécifique; et dans le cadre d'une cérémonie vaudou, les Cassés sont multi-fonctionnels.

D'abord on croit que cette puissante brisure rythmique est encore une façon d'exciter les danseurs et les loas. Puis, les Cassés sont employés par le maître-tambour pour passer d'un message-tambour à un autre, sans interruption, et dans ce cas, le Cassé est généralement encadré d'une forme quelconque de roulement, spécifique au rythme joué. Le minokan, série continue de rythmes en contexte bien particulier, fait usage de ce principe. En voici un exemple typique:

Rythme 1/Cassé 1/Roulement/Cassé 2/Roulement/

Rythme 2/Roulement/Cassé 3/Rythme 3...

La fonction principale du rythme Cassé est d'indiquer aux danseurs un changement dans les mouvements: par exemple, après avoir dansé sur place, le cassé "dit» de commencer à "voyager», ce qui veut dire, tourner autour du poteau-mitan (poteau central dans l'aire de déroulement de la cérémonie) en signe de respect envers ce poteau qui symbolise un des chemins que peut prendre un loa rendant visite à une cérémonie. Ou encore, dans une série de quatre battements, les trois premiers peuvent par exemple indiquer un mouvement vers la gauche et le quatrième, un mouvement vers la droite. A l'arrêt ou en mouvement, les danseurs attendent le signal des tambours avant de procéder aux changements.

Examinions de plus près quelques-uns des éléments essentiels à la constructions d'un rythme Cassé, d'une part du point de vue de la sélection des sons proprement dits (a) et d'autre part du point de vue de l'organisation structurelle (b).

a) Les sons utilisés dans le tambourinage vaudou se regroupent naturellement en quelques catégories simples que la langue créole a précisément dénommées, comme on peut le voir dans l'exemple 4. Il est essentiel d'en discuter parce que les facteurs acoustiques sont déterminants pour la venue des Loas; de ces facteurs dépend donc la construction même des tambours. Rappelons qu'un peu plus haut, il a été dit que le "timbre" des tambours sert d'indice pour reconnaître de quel rituel il s'agit. Les sons dans le rituel Rada résonnent vraiment différemment de ceux des autres rituels même s'ils portent un nom identique et sont produits avec les mêmes techniques de main et de baguette. 


\begin{tabular}{|c|c|c|c|}
\hline Nom du son & $\begin{array}{l}\text { Hauteur } \\
\text { relative }\end{array}$ & $\begin{array}{l}\text { Lieu de } \\
\text { production } \\
\text { du son }\end{array}$ & Position/main/baguette \\
\hline $\begin{array}{l}\text { "Clair"/ } \\
\text { "ouvert" }\end{array}$ & 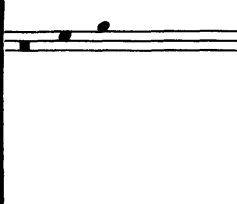 & $\left\{\begin{array}{l}\text { milieu-bord } \\
\text { milieu-bord }\end{array}\right.$ & $\begin{array}{l}\text { main à plat, doigts } \\
\text { ensembles, articulation } \\
\text { main-doigts au bord } \\
\text { baguette }\end{array}$ \\
\hline "Basse" & & milieu & $\begin{array}{l}\text { main creuse } \\
\text { sans baguette }\end{array}$ \\
\hline "Etouffé" & $\Longrightarrow 0$ & milieu-bord & $\begin{array}{l}\text { comme pour son 'clair' } \\
\text { mais assourdi par bord } \\
\text { des doigts } \\
\text { sans baguette }\end{array}$ \\
\hline "Pianissimo" & $\Leftrightarrow$ & bord & $\begin{array}{l}\text { bout de } 1 \text { ou } 2 \text { doigts } \\
\text { sans baguette }\end{array}$ \\
\hline "Siré" & $\overline{10}$ & bord à milieu & $\begin{array}{l}\text { bout du majeur glissé } \\
\text { sans baguette }\end{array}$ \\
\hline Coudé * & 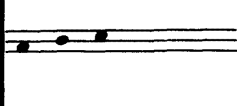 & milieu + bord & $\begin{array}{l}\text { coude + main ouverte } \\
\text { ou baguette }\end{array}$ \\
\hline “Cassé” & & $\underset{\text { milieu }}{\text { milieu-bord + }}$ & $\begin{array}{l}\text { bout des doigts ou } \\
\text { baguette + main gauche } \\
\text { ouverte laissée sur } \\
\text { tambour }\end{array}$ \\
\hline Claqué * & & $\begin{array}{l}\text { milieu + } \\
\text { dessous } \\
\text { du tambour }\end{array}$ & $\begin{array}{l}\text { main ouverte }+ \\
\text { baguette }\end{array}$ \\
\hline \multicolumn{4}{|c|}{$\begin{array}{l}\text { "Les deux noms marqués d'une étoile n'ont pas de dénomination spéci- } \\
\text { fique. C'est pourquoi, inspirés par la manière de les produire, Georges } \\
\text { Rodriguez et moi-même avons convenu de les nommer ainsi. }\end{array}$} \\
\hline
\end{tabular}


Dans ce tableau, les hauteurs sont relatives; toutefois, les hauteurs des sons "pianissimo" et des sons "étouffés" sont perçues en relation avec les sons plus forts qui les entourent dans le courant d'un rythme. Ainsi l'illusion d'une variété de hauteurs qui ne reflète en rien leur degré d'importance. Le son "cassé » caractérise les rythmes Cassés bien qu'on le trouve aussi dans les rythmes de base; d'ailleurs, le mot créole "cassé » a sans doute donné son nom aux sections rythmiques dans lesquelles prédomine ce son.

En général, le rythme de base fait ample usage de tous les sons représentés dans l'exemple 4 tandis que la section Cassé insiste sur les sons "cassé " et "claqué", ses autres sons étant surtout répartis entre les sons "basse» et "clair»; mais ces derniers sont joués si fort qu'ils peuvent sembler tout à fait différents des sons "basse" et "clair» des rythmes de base. L'exemple suivant offre une transcription du rythme Yanvalou quatre et de sa section Cassé.

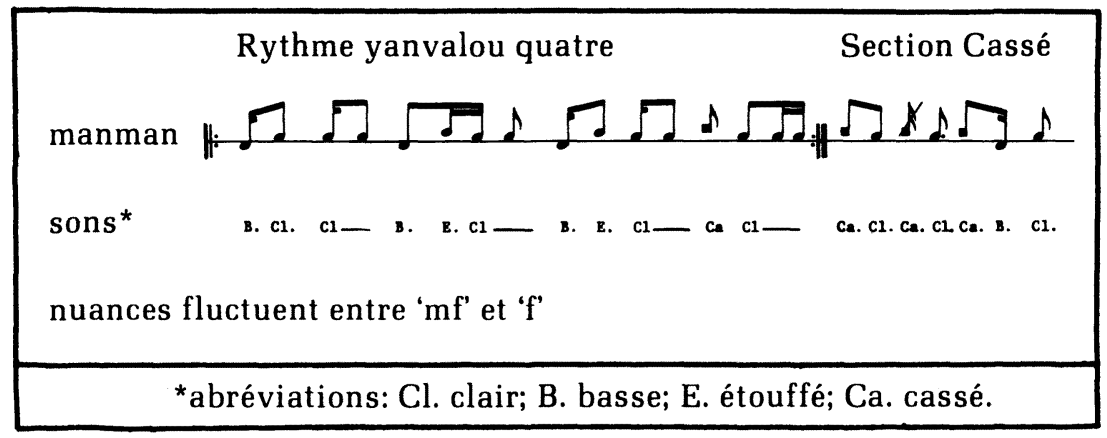

Exemple 5

Dans cet exemple, l'intensité est le facteur de différenciation le plus évident; de plus, l'ambitus privilégie les hauteurs extrêmes du tambour, lesquelles contribuent à capter l'attention de l'oreille.

b) Les sections Cassé diffèrent l'une de l'autre dans leur organisation des durées. Malgré le fait qu'on ait l'impression d'un déplacement d'accent métrique, ils demeurent identiques à leur ry thme de base quant à la longueur des segments. Ensuite, les Cassés sont en principe construits sur trois séries de quatre battements chacun; mais la série peut être précédée d'un fragment de Cassé pour indiquer que justement, il est temps de jouer 
un Cassé, comme on peut le voir dans l'exemple suivant qui met en paradigme les Cassés de quatre des rythmes de la famille Yanvalou:

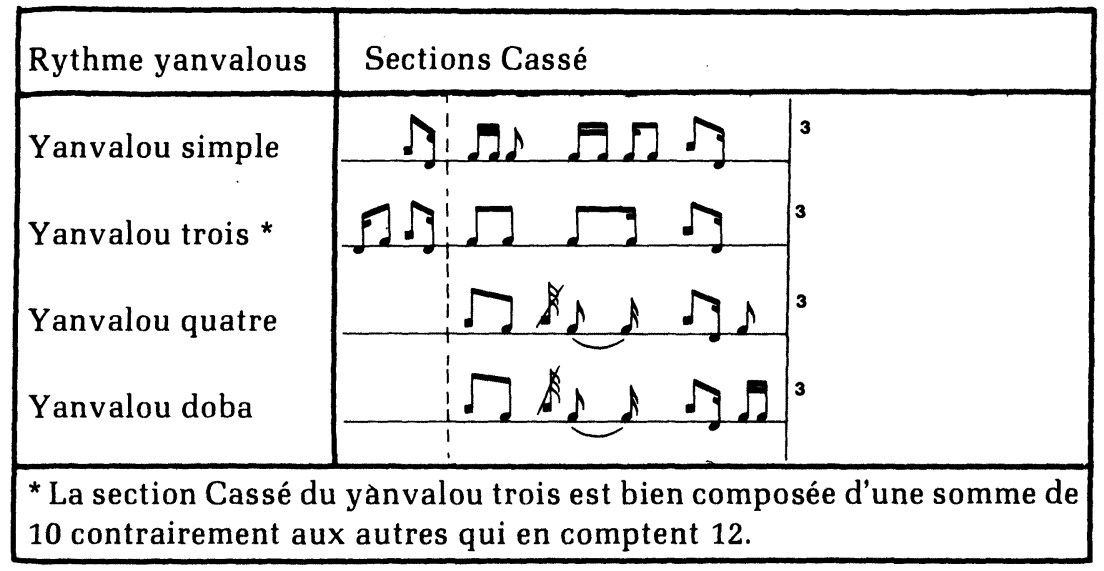

Exemple 6

Voyons maintenant ce qui se passe dans les Cassés de trois des rythmes de la famille Mahi.

Le principe de série répétée de battements sur lequel avait tant insisté le tambourineur se vérifie dans la famille Yanvalou mais les exemples de la famille Mahi les contredisent nettement; sans doute peut-on supposer qu'interviennent ici d'autres facteurs qui influencent la construction d'un Cassé, facteurs qui dépassent le cadre d'un simple signal aux danseurs.

Même si on évite d'aller trop loin dans les détails, on est frappé dans les exemples 6 et 7 de la similarité de construction des Cassés et on se demande si les danseurs perçoivent vraiment la différence entre, par exemple, le Cassé du Yanvalou quatre et celui du Yanvalou doba, ou bien s'ils connaissent automatiquement de quel contexte il s'agit, puisqu'ils connaissent par coeur les cérémonies. On nous a affirmé qu'ils étaient effectivement sensibles à ces changements, même lorsqu'ils nous apparaissent minimes, mais nous pensons que le point a encore besoin d'être vérifié.

Les Cassés Mahi sont construits à partir de trois éléments principaux:

La présence du son "cassé», suivi rapidement d'un son 


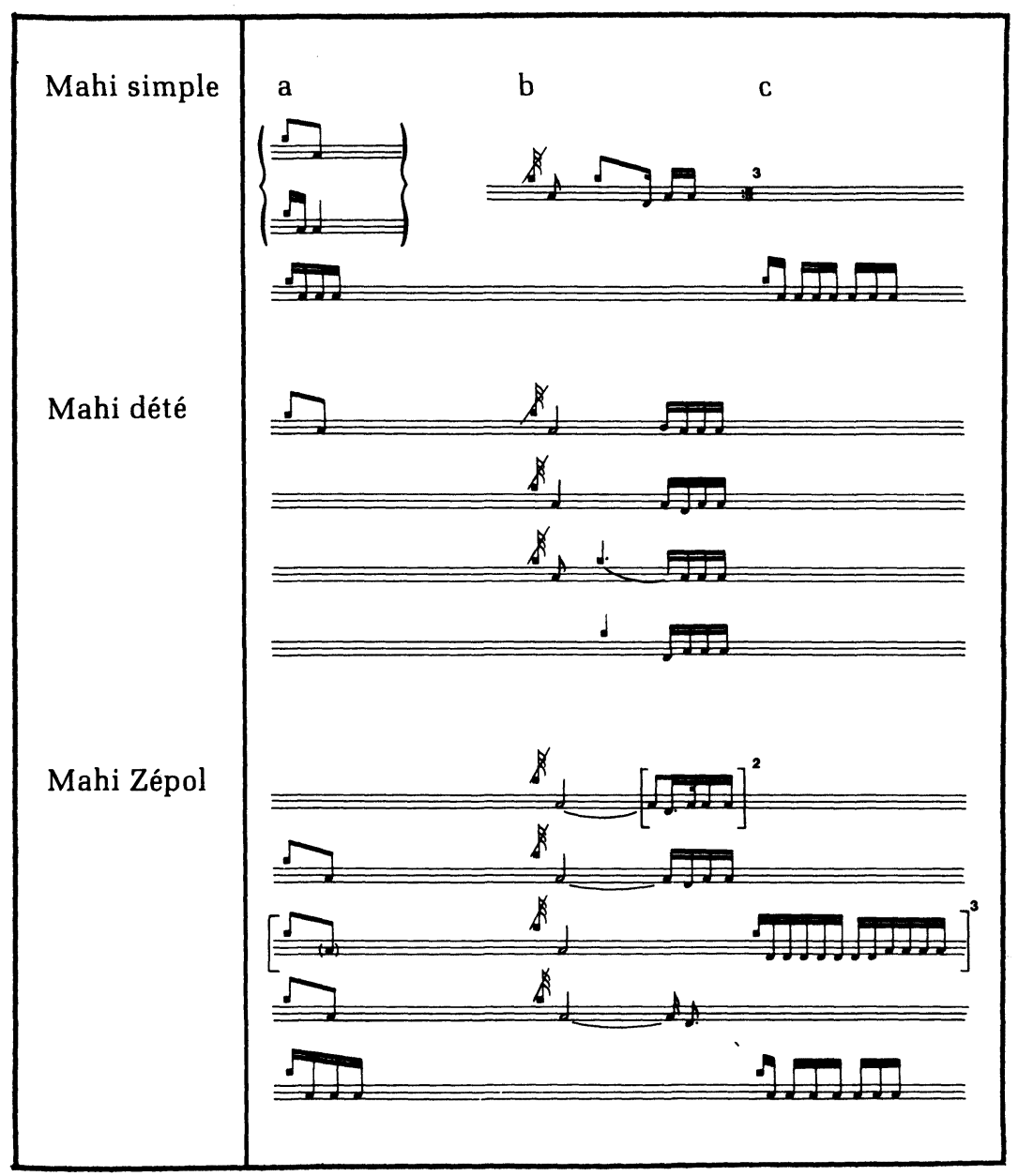

Exemple 7 
"clair» tenu ou répété; systématiquement aussi, il incorpore les roulements utilisant aussi le son "cassé». La colonne "c " montre le roulement tel qu'il se joue comme roulement tandis que les colonnes " $a$ " et « $b$ " ne révèlent que le contour caractéristique du roulement comme élément de base: grand saut vers le bas en " a » et répétition de note en " b ». Cette description de la construction d'un Cassé devient significative à la lumière du comportement formel des autres rythmes et de leurs Cassés. Tous semblent sujets à des emprunts considérables, ce qui cause un degré de confusion appréciable quant à la pertinence des regroupements classificatoires de classes rythmiques en relation avec les classes ou familles de danses, de loas et de circonstances rituelles.

En réfléchissant à tout ce qui a été dit jusqu'ici, il semble que nous ayions dû tenir compte d'aspects multiples du tambourinage vaudou pour comprendre deux cas relativement simples de signaux rythmiques. Il était d'autant plus réaliste d'avoir choisi de discuter d'exemples simples, mais nous avions aussi choisi ces deux cas, parce que chacun représentait différents niveaux de transmission de message.

Le Kata, au niveau de la liturgie totale, devient une sorte de représentation abstraite d'un rituel spécifique; discret parce que dispersé à travers un fouilli de sons, il s'adresse non moins précisément aux humains et aux loas. Le Cassé, lui, témoigne d'un symbolisme plus concret: il donne des indications précises aux danseurs d'une manière forte et évidente.

Une analyse pour être pertinente doit tenir compte des différences de niveaux de signification et du poids sémantique de chaque unité de signification. L'analyse doit aussi tenir compte de la quantité et de l'efficacité relative de signes qui fonctionnent simultanément, c'est-à-dire du système de signes dont une unité fait partie. Par exemple, on peut affirmer que les tambours ont quelque chose à dire, mais en s'adressant aux loas, les signaux de tambour devraient toujours être mis en parallèle avec les dessins sacrés (les vévés), sans lesquels jamais les loas ne viendraient. Prenons un exemple d'un ordre différent: la métrique n'est pas uniquement déterminée par le contexte du rythme de base; souvent, dans des rythmes où la répétition de motifs ne correspond pas à la longueur des mesures, on hésite à y entendre deux, quatre ou huit battements et on doit renvoyer la décision à la répétition des motifs gestuels correspondants; et encore, que faut-il penser du fait que les métriques gestuelles et 
de tambour ne correspondent pas nécessairement à la métrique de la partie vocale?

Le Kata fonctionne indépendamment de tout ceci, mais non le Cassé. Le Kata est un symbole parmi tant de symboles aux usages multivalents. Le Cassé est un symbole solitaire, ne transmettant qu'un seul message à la fois. Il reste à trouver comment une analyse peut rendre compte de tous ces phénomènes à la fois. Chose certaine, le fait que la rythmique soit un langage dans les rituels vaudous n'est pas un phénomène isolé. Évidemment, le vaudou haïtien a des origines africaines qu'on retrouve aisément, mais son contenu symbolique ne résulte pas simplement d'une parenté géographique. Nous croyons vraiment avoir touché du doigt (ou plutôt de l'oreille) un mode de fonctionnement des unités musicales en général. Différents pays, différents groupements humains, différents besoins créent des contextes symboliques différents. Il appartient aux musicologues et aux autres chercheurs non seulement d'être sensibles à ces différences mais d'en reconnaître les principes fondamentaux, communs à tous les êtres humains. 


\section{NOTES}

1. Les recherches dont rend compte le présent article ont été rendues possibles grâce à une subvention de voyage en Haïti, octroyée par la Faculté des Études Supérieures de l'Université de Montréal (1979) et grâce à une subvention de recherche du fonds Alma Mater de la même Université (1980). Une première version de cet article a été présentée au Congrès annuel de la Society for Ethnomusicology, à Bloomington, Indiana (Novembre 1980).

2. Une abondante littérature décrit les personnages, l'attirail et les lieux rituels. Comme ils ne font pas l'objet de la présente discussion, nous renvoyons le lecteur à l'excellent ouvrage d'Alfred Métraux (1958).

3. Les tambours changent de nom et de forme dans les différents rituels mais non le ogan (cloche ou lamelle de fer épaisse).

4. Ces enregistrements ont été réalisés dans des conditions idéales: la Faculté de musique de l'Université de Montréal a mis à notre disposition son Studio de Musique Electro-acoustique ainsi que les services de techniciens compétents. Les sessions d'enregistrement ont été réparties sur plusieurs mois en 1979-1980.

5. Dans un avenir prochain, nous serons en mesure de substituer aux rythmes de base du tambour-maitre, les variantes habituelles ou occasionnelles, ce qui nous donnera accès aux règles de transformation rythmique. Nous pourrons alors étudier la constance d'un message à travers la fluctuation de son expression.

6. Un jour, nous compléterons la série en ajoutant sur vidéo, les mouvements de danse, eux aussi spécifiques des différents rythmes. Ces enregistrements (et par conséquent, leur transcription) auraient été impensables sans le talent et l'inlassable patience de Georges Rodriguez qui a si généreusement partagé avec nous sa compétence en matière d'interprétation des données ethnographiques et de la rythmique haïtienne. En d'autres termes, nous avons eu l'occasion à faire connaissance avec les données ethnographiques, à Montréal même. 
AROM, S.

\section{RÉFÉRENCES}

1976: "The Use of Play-Back Techniques in the Study of Oral Polyphonies », Ethnomusicology, XX/3, 453-518.

COURLANDER, $\mathrm{H}$.

1960 : The Drum and the Hoe. Berkeley: University of California Press.

JONES, A.M.

1959 : Studies in African Music. London: Oxford University Press. MÉTRAUX, A.

1958 : Le Vaudou Haïtien, 2ème éd. Paris: Gallimard. 